\title{
The Effects of PMM2-CDG-Causing Mutations on the Folding, Activity, and Stability of the PMM2 Protein
}

Patricia Yuste-Checa, Alejandra Gámez, Sandra Brasil, Lourdes R. Desviat, Magdalena Ugarte, Celia Pérez-Cerdá, and Belén Pérez*

\begin{abstract}
Centro de Diagnóstico de Enfermedades Moleculares, Centro de Biología Molecular-SO UAM-CSIC, Universidad Autónoma de Madrid, Campus de Cantoblanco, 28049 Madrid/Centro de Investigación Biomédica en Red de Enfermedades Raras (CIBERER), Instituto de Investigación Sanitaria IdiPaZ, Madrid, Spain
\end{abstract}

Communicated by Jan P. Kraus

Received 7 January 2015; accepted revised manuscript 19 May 2015.

Published online 25 May 2015 in Wiley Online Library (www.wiley.com/humanmutation). DOI: 10.1002/humu.22817

\begin{abstract}
Congenital disorder of glycosylation type Ia (PMM2-CDG), the most common form of CDG, is caused by mutations in the PMM2 gene that reduce phosphomannomutase 2 (PMM2) activity. No curative treatment is available. The present work describes the functional analysis of nine human PMM2 mutant proteins frequently found in PMM2-CDG patients and also two murine Pmm2 mutations carried by the unique PMM2. CDG mouse model described to overcome embryonic lethality. The effects of the mutations on PMM2/Pmm2 stability, oligomerization, and enzyme activity were explored in an optimized bacterial system. The mutant proteins were associated with an enzymatic activity of up to $47.3 \%$ as compared with wild type (WT). Stability analysis performed using differential scanning fluorimetry and a bacterial transcription-translation-coupled system allowed the identification of several destabilizing mutations (p.V44A, p.D65Y, p.R123Q, p.R141H, p.R162W, p.F207S, p.T237M, p.C241S). Exclusion chromatography identified one mutation, p.P113L, that affected dimer interaction. Expression analysis of the p.V44A, p.D65Y, p.R162W, and p.T237M mutations in a eukaryotic expression system under permissive folding conditions showed the possibility of recovering their associated PMM2 activity. Together, the results suggest that some loss-of-function mutations detected in PMM2-CDG patients could be destabilizing, and therefore PMM2 activity could be, in certain cases, rescuable via the use of synergetic proteostasis modulators and/or chaperones.

Hum Mutat 36:851-860, 2015. (C) 2015 Wiley Periodicals, Inc.
\end{abstract}

KEY WORDS: phosphomannomutase 2; PMM2; congenital disorders of glycosylation; PMM2-CDG; protein stability

Additional Supporting Information may be found in the online version of this article. Contract grant sponsors: Fundación Ramon Areces grant; MINECO grants IPT-20120561-010000 and PI11/01250; Ramon y Cajal grant from the Ministerio de Ciencia y Tecnología.

*Correspondence to: Belén Pérez, Centro de Diagnóstico de Enfermedades Moleculares, Centro de Biología Molecular UAM-CSIC, Universidad Autónoma Madrid, Madrid, Spain. E-mail: bperez@cbm.csic.es

\section{Introduction}

Over 70 diseases have been identified as congenital disorders of glycosylation (CDG) [Hennet, 2012; Foulquier et al., 2012; Freeze et al., 2014]-inherited metabolic disorders caused by defects in the biosynthesis of glycoconjugates. The vast majority involve protein hypoglycosylation defects, but in recent years, several defects of lipid glycosylation have also been identified [Jaeken and Matthijs, 2007; Freeze et al., 2014]. Defects in the N-glycosylation pathway account for most protein glycosylation disorders; the remainder involve the $\mathrm{O}$-glycosylation pathway or both $\mathrm{N}$ - and $\mathrm{O}$-glycosylation together. No C-glycosylation defects are known.

Congenital disorder of glycosylation type Ia (PMM2-CDG; MIM \#212065) is caused by mutations in the PMM2 gene (MIM \#601785) [Matthijs et al., 1997] that reduce the activity of their product enzyme phosphomannomutase 2 (PMM2) [Van Schaftingen and Jaeken, 1995]. No strong genotype-phenotype correlation exists [Grunewald et al., 2001] that might suggest the involvement of any other gene, and as it has been suggested for an ALG6 polymorphism, it is twice as common in severely affected PMM2-CDG patients than in patients classified as moderate/mild [Westphal et al., 2002]. PMM2, a homodimeric cytosolic protein, plays an important role in $\mathrm{N}$-glycosylation by competing with phosphomannose isomerase for mannose-6-phosphate, converting it into mannose-1-phosphate for entry into the $\mathrm{N}$-glycosylation pathway. Over 1,000 cases of PMM2CDG [Cylwik et al., 2013] have been reported, involving $116 \mathrm{mu}-$ tations, most of them $(83.6 \%)$ missense changes (HGMD ${ }^{\circledR}$ professional release 2014.3, http://www.hgmd.cf.ac.uk/ac/index.php) [Stenson et al., 2014].Patients with this type of changes are usually compound heterozygous for two different missense mutations. One severe mutation, p.R141H, is particularly common in every analyzed cohort of patients, but in spite of the high number of detected carriers, no homozygous patients have been reported [Briones et al., 2002; Kjaergaard et al., 1998; Le Bizec et al., 2005; Matthijs et al., 1998].

The hypoglycosylation of glycoproteins and other glycoconjugates affects the functioning of multiple body systems. Currently, there is no curative treatment for patients with PMM2-CDG; most of those affected suffer motor problems and mental retardation [Freeze, 2006], and their prognosis can be extremely poor. However, patients with very mild disease have been reported [Giurgea et al., 2005], some of whom show under $25 \%$ of normal enzyme activity. Indeed, their heterozygous parents, with $50 \%$ activity, remain asymptomatic [Freeze, 2009]. Such a high symptomatic threshold suggests that increasing the PMM2 activity of patients with PMM2CDG might be very beneficial. Certainly, it has been widely observed 
that patients with high residual activity tend to have milder phenotypes [Westphal et al., 2002].

Some therapeutic approaches for PMM2-CDG have been proposed. MPI enzyme inhibition combined with mannose administration or bypassing the defect by membrane permeable mannose-1-P have been suggested [Freeze, 2009]. However, sugar supplementation presents some important hurdles such as the fact that mannose metabolic flux should be manipulated cautiously [Cline et al., 2012; Sharma et al., 2014a; Sharma et al., 2014b] and the need to study and develop a delivery system to the cell for mannose-1-phosphate [Eklund et al., 2005; Freeze, 2009; Hardre et al., 2007]. Other proposed therapies could be enzyme replacement or gene therapy, although the main problem would be the intracellular loading or the research on gene delivery safety. Over the last decade, pharmacological chaperones have emerged as a novel therapeutic tool to rescue misfolded proteins by stimulating and preserving their correct folding [Martinez et al., 2008; Muntau et al., 2014].

The aim of the present work was to search for candidate mutations that might be rescued by such treatment. The PMM2 protein expression system was therefore optimized in a bacterial system, allowing a number of human PMM2 mutant proteins detected among Spanish PMM2-CDG patients [Perez et al., 2011]—p.V44A (c.131T >C), p.D65Y (c.193G>T), p.P113L (c.338C >T), p.R123Q (c.368G>A), p.R141H (c.422G>A), p.R162W (c.484C >T), p.F207S (c.620T >C), p.T237M (c.710C > T), and p.C241S (c.722G $>C$ ) - to be purified in their native states. The stability and activity of some of these proteins were then examined in a eukaryotic system.

Two murine Pmm2 mutant proteins, p.F118L (c.354C $>\mathrm{G}$ ) and p.R137H (c.310_311delinsAC.; analogous to the p.R141H mutant in humans), were also expressed in a bacterial system to investigate the molecular mechanism underlying the benefit of prenatal mannose supplementation in dams at risk of having pups carrying these two mutations [Schneider et al., 2012]. The study of these two mutations would allow to test the feasibility of the PMM2-CDG murine model as a mean of investigating proteostasis and chaperone therapies' strategies.

Together, the results suggest that loss-of-function mutations are destabilizing, but that PMM2 activity may sometimes be rescuable.

\section{Materials and Methods}

\section{Wild-Type PMM2 and Mutant Gene Expression}

The expression plasmid pDEST17-18 encoding human PMM2 (NM_000303.2) plus an N-terminal $\mathrm{His}_{6}$-tag, and pReceiver-B01 encoding mouse Pmm2 (NM_016881.2) plus an N-terminal His ${ }_{6}$ tag (Source BioScience), were used to transform E. coli strain BL21Star ${ }^{\mathrm{TM}}$ DE3 One Shot Cells (Invitrogen, Carlsbad, CA, USA). $P M M 2$ mutations were introduced by site-directed mutagenesis using the QuikChange Mutagenesis Kit (Stratagene, Cedar Drive, TX, USA) and specially designed primers. All products were verified by DNA sequencing. Mutation nomenclature is based on cDNA sequence (PMM2 NM_000303.2; Pmm2 NM_016881.2) using +1 as the A of the ATG translation initiation codon in the reference sequence, considering the initiation codon as codon 1 . For protein expression, bacteria were grown in modified TYM medium (Studier autoinduction medium [Studier, 2005] with $\mathrm{N}-\mathrm{Z}$ amine replaced by tryptone) containing $25 \mu \mathrm{g} / \mathrm{ml}$ zeocine over night at $37^{\circ} \mathrm{C}$. Cells were harvested by centrifugation, resuspended in $20 \mathrm{mM}$ HEPES, $25 \mathrm{mM}$ $\mathrm{KCl}, 1 \mathrm{mM}$ DTT, pH 7, and $1 \times$ Complete Mini, EDTA-free Protease Inhibitor Cocktail (Roche Applied Biosciences, Indianapolis, IN, USA), lysed by sonication, and then centrifuged at $4^{\circ} \mathrm{C}$. Protein concentrations were determined following the Bradford method [Bradford, 1976] using Bio-Rad Protein Assay Reagent (Bio-Rad, Munchen, Germany). This crude soluble cell extract was used for protein activity assays, western blotting, and protein purification.

\section{Protein Purification}

Protein purification was performed using the ÄKTA Prime System (GE healthcare, Buckinghamshire, UK) at $4^{\circ} \mathrm{C}$. The crude extract was loaded onto a HisTrap ${ }^{\text {TM }}$ High Performance affinity column (GE Healthcare) equilibrated with $10 \mathrm{mM}$ imidazole, $0.5 \mathrm{M} \mathrm{NaCl}$, $20 \mathrm{mM}$ sodium phosphate, $\mathrm{pH}$ 7.4, and eluted with an imidazole gradient from $10 \mathrm{mM}$ to $1 \mathrm{M}$. The eluted protein fractions were pooled and loaded into a Superdex 200 HiLoad 16/60 size exclusion chromatography column (GE healthcare). The elution fraction corresponding to dimeric PMM2 was recovered and stored for further experiments. The pure protein concentration was estimated by measuring the absorbance at $280 \mathrm{~nm}$ in a Nanodrop spectrophotometer (Thermo Scientific, Waltham, MA, USA) using the theoretical molar extinction coefficient estimated from the amino acid composition of HisPMM2 $\left(23,755 \mathrm{M}^{-1} / \mathrm{cm}\right)$.

\section{Western Blotting}

Samples were subjected to electrophoresis in $10 \%$ NuPAGE $^{\circledR}$ BisTris Precast Gels (Invitrogen). ProSieve ${ }^{\circledR}$ Color Protein Markers (Lonza, Basel, Switzerland) were used as molecular weight markers. Proteins were transferred to a nitrocellulose membrane using the iBlot ${ }^{\circledR}$ Dry Blotting System (Invitrogen). Membranes were blocked for at least $1 \mathrm{hr}$ with $0.05 \%$ PBS-Tween and 5\% low-fat milk. Immunodetection was performed using primary mouse polyclonal antibodies to PMM2 protein (A01; Abnova, Heidelberg, Germany), polyhistidine Sigma-Aldrich (St. Louis, MO, USA) and tubulin (Sigma-Aldrich). Conjugated goat antimouse immunoglobulin G horseradish peroxidase (Santa Cruz Biotechnology, Santa Cruz, CA, USA) was used as the secondary antibody. The Enhanced Chemiluminescence System (GE healthcare) was used as the detection method. Relative amounts of protein were determined by densitometry using a Bio-Rad GS710 Calibrated Imaging Densitometer running Quantity One 4.3.1 software (Bio-Rad).

\section{Cell Culture}

Patient-derived fibroblasts (P1-P4) were grown from patient skin biopsies (taken with informed consent) following standard conditions in minimal essential medium supplemented with $1 \%$ glutamine, $10 \%$ fetal calf serum, and antibiotics. These cells were then immortalized using pBABE-puro containing SV40 DNA sequences (courtesy of Dr. J. A. Enríquez). The GM08680 cell line (Coriell Institute for Medical Research, NIGMS Human Genetic Cell Repository, Camden, New Jersey) was used as a control.

\section{PMM2 Activity Assay}

The PMM2 activity of the bacterial extract and pure protein was assayed using the method of Van Schaftingen and Jaeken [Van Schaftingen and Jaeken, 1995], as modified by de Koning et al. [1998], but doubling the reagent concentrations. Assays were performed using $50 \mathrm{ng}$ of nanodrop-measured pure dimeric protein, or $300 \mathrm{ng}$ of bacterial extract as measured by the Bio-Rad Protein 
Assay (Bio-Rad). The reaction is measured by NADPH presence at $340 \mathrm{~nm}$

To improve the reproducibility and sensitivity of the results for PMM2 activity in patient-derived fibroblasts, changes were introduced to the classic activity assay, the lysis buffer, in order to obtain higher amount of protein from less amount of cells, and the way in which the product signal was measured, using a fluorimetric kit and finally measuring the NADPH production by fluorescence at $540 / 590 \mathrm{~nm}$. These modifications increased the reproducibility and sensitivity of the whole method and allowed the use of 96-well plates, rendering the process more suited to high-throughput evaluation.

Some 15,000 cells per well were seeded in a 96-well plate and lysed using Complete Lysis M reagent (Roche Applied Biosciences). PMM2 activity was assayed using the same method as for the bacterial extracts and pure protein, incubating the plates for $45 \mathrm{~min}$, or $4 \mathrm{hr}$ for the P3 and $\mathrm{P} 4$ cell line. The NADPH production signal was increased using the Amplite Fluorimetric NADPH assay Kit (AAT Bioquest, Sunnyvale, CA, USA). Protein extracts from fibroblasts were measured using the Bio-Rad Protein Assay (Bio-Rad).

\section{Differential Scanning Fluorimetry}

The stability of purified PMM2 was assessed by differential scanning fluorimetry (DSF) [Niesen et al., 2007], monitoring its thermal denaturation in the presence of the extrinsic fluorescent probe SYPRO Orange (Sigma-Aldrich). Final volumes of $50 \mu \mathrm{l}$ containing $0.075 \mathrm{mg} / \mathrm{ml}$ of pure PMM2 in $20 \mathrm{mM} \mathrm{Na}$-HEPES pH 7.0, 200 $\mathrm{mM} \mathrm{NaCl}$, and 5x SYPRO Orange were dispensed into LightCycler480 Multiwell 96-well PCR-plates (Roche Applied Biosciences). These were then loaded into a Light Cycler 480 (Roche Applied Biosciences) for thermal denaturation. Unfolding curves were recorded from $20^{\circ} \mathrm{C}$ to $85^{\circ} \mathrm{C}$ at a scan rate of $2^{\circ} \mathrm{C} / \mathrm{min}$. The increase in SYPRO Orange fluorescence intensity associated with protein unfolding $\left(\lambda_{\text {excitation }}=465 \mathrm{~nm} ; \lambda_{\text {emission }}=580 \mathrm{~nm}\right)$ was monitored as a measure of thermal denaturation. The experimental unfolding curves were then smoothed, normalized, and analyzed using in-house software, fitting the data to sigmoid curves using an asymmetry parameter [Pey et al., 2008]. The midpoint melting temperature $\left(T_{\mathrm{m}}\right)$ was calculated as that at which half of the protein was in the unfolded state.

\section{Thermal Stability Profiles}

Dimeric PMM2 used for thermal stability analysis was produced by the RTS 100 E.coli HY kit (5 PRIME, Hilden, Germany) transcription-translation-coupled system, following the manufacturer's recommendations. After $30 \mathrm{~min}$ of incubation at $30^{\circ} \mathrm{C}$ to produce human PMM2, or 60 min to generate mouse Pmm2, the reaction was stopped by adding $1 \mu \mathrm{l}$ of DNase and RNase $(1 \mathrm{mg} / \mathrm{ml})$. The whole reaction was incubated at $37^{\circ} \mathrm{C}$, and $1.5 \mu \mathrm{l}$ aliquots removed at different times. Samples were examined by western blotting as described above.

\section{FoldX Prediction}

FoldX is a computer algorithm that provides a quantitative estimate of the importance of the interactions contributing to the stability of proteins and protein complexes. It uses a full atomic description of the structure of proteins. The different energy terms taken into account were weighted using empirical data obtained from protein-engineering experiments. For a detailed explanation of the FoldX force field, see Schymkowitz et al. [2005a, 2005b] and information on the FoldX Web server (http://foldx.crg.es).

To study the effects of PMM2 mutations on protein stability, the $<$ BuildModel $>$ command in FoldX v.3.0 and the PMM2 PDB file 2AMY (PMM2 monomer), previously programmed using the RepairPDB command, was used. The effect of the mutation was computed by subtracting the energy of the WT from that of the mutant (positive numbers mean less stability). The difference in energy between the mutation and the WT reference is provided in kilocalories per mole.

\section{Lentiviral Constructs}

The full-length ORF of human PMM2 was cloned into the mammalian lentiviral plasmid pReceiver-Lv101 (EX-M0134-Lv101), which contains the FLAG tag preceding the multiple cloning site (GeneCopoeia, Rockville, MD). Lentiviral stock production and fibroblast infection were performed as described elsewhere [Richard et al., 2013]. Efficiently infected fibroblasts were selected by Geneticin treatment.

\section{Statistical Analysis}

Statistical analyses were performed using IBM SPSS Statistics 21 software for Windows. One-way ANOVA followed by a Bonferroni post hoc test was used to compare PMM2 activity and stability as measured by DSF and the degradation time course assays.

\section{Results}

Human His $_{6}-\mathrm{PMM} 2$ WT and p.V44A, p.D65Y, p.P113L, p.R123Q, p.R141H, p.R162W, p.F207S, p.T237M, and p.C241S, and mouse $\mathrm{His}_{6}-\mathrm{Pmm} 2 \mathrm{WT}$, p.F118L and p.R137H, were expressed in the above-mentioned prokaryotic system. Western blotting performed with the soluble protein extracts showed the amount of PMM2 to be reduced for all mutants compared with WT, except for the mouse p.F118L mutant. No soluble protein was detected for the p.F207S mutant (Supp. Fig. S1). Using this well-established system, we have studied the effect of these mutations on the oligomerization profile, activity, and stability of the PMM2 protein.

\section{Oligomerization Profiles}

The different oligomeric states of the mutant proteins were analyzed by size-exclusion chromatography. $\mathrm{His}_{6} \mathrm{PMM} 2 \mathrm{WT}$ and mutants were purified by affinity chromatography and then loaded onto a size-exclusion chromatography column to obtain an oligomerization profile. For all human PMM2 mutants, the amount of pure protein obtained after purification was $<30 \%$ that obtained with the WT (starting with the same amount of bacterial culture) (Table 1]. Exclusion chromatography analysis revealed three oligomerization patterns: (1) the mutants p.V44A, p.D65Y, p.R162W, and p.T237M showed oligomeric profiles similar to that of the WT, with abundance of the dimeric fraction; (2) p.R123Q, p.R141H and p.C241S showed profiles with a predominant aggregate fraction; and (3) mutant p.P113L returned no oligomeric profile (Fig. 1A and C; Table 1). Mutation p.P113L acts at the interface between the dimer subunits and probably affects dimerization [Silvaggi et al., 2006; Vega et al., 2011]. No p.F207S protein could be purified given the lack of soluble protein expression in the prokaryotic system (Supp. Fig. S1). 


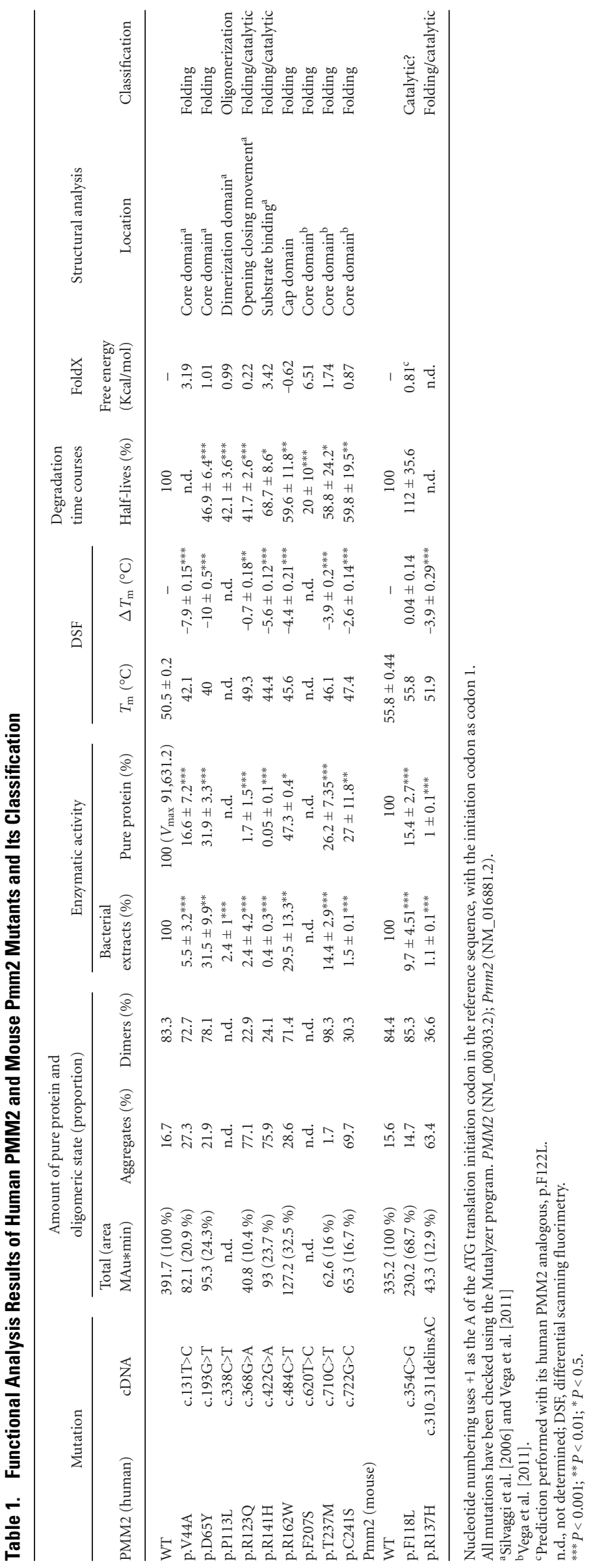


A
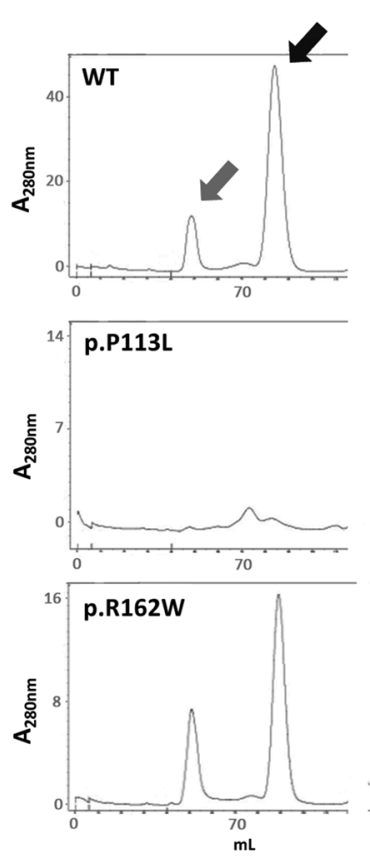
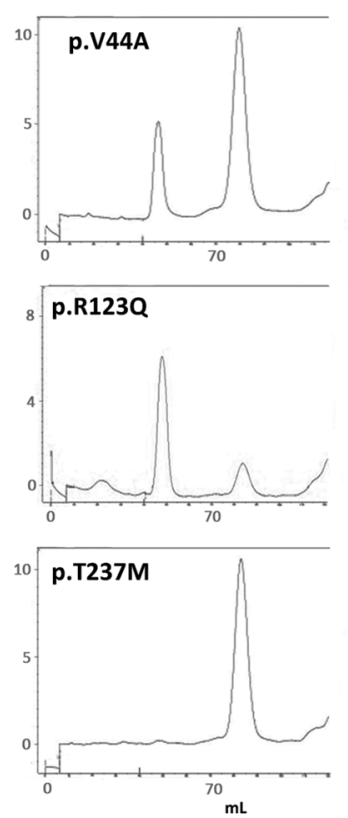

B
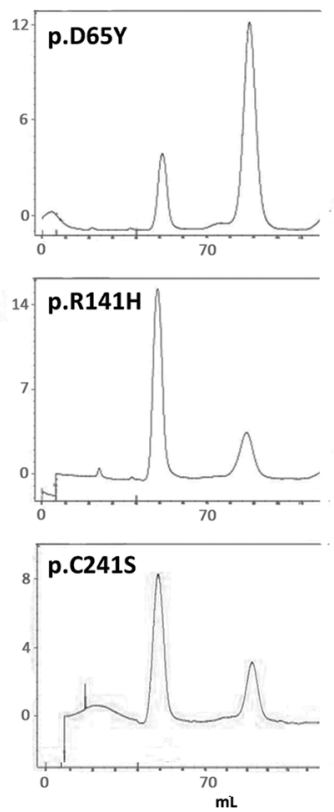
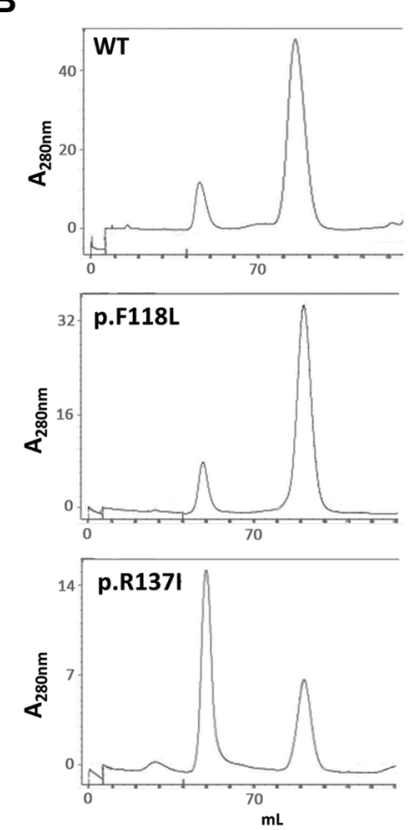

C

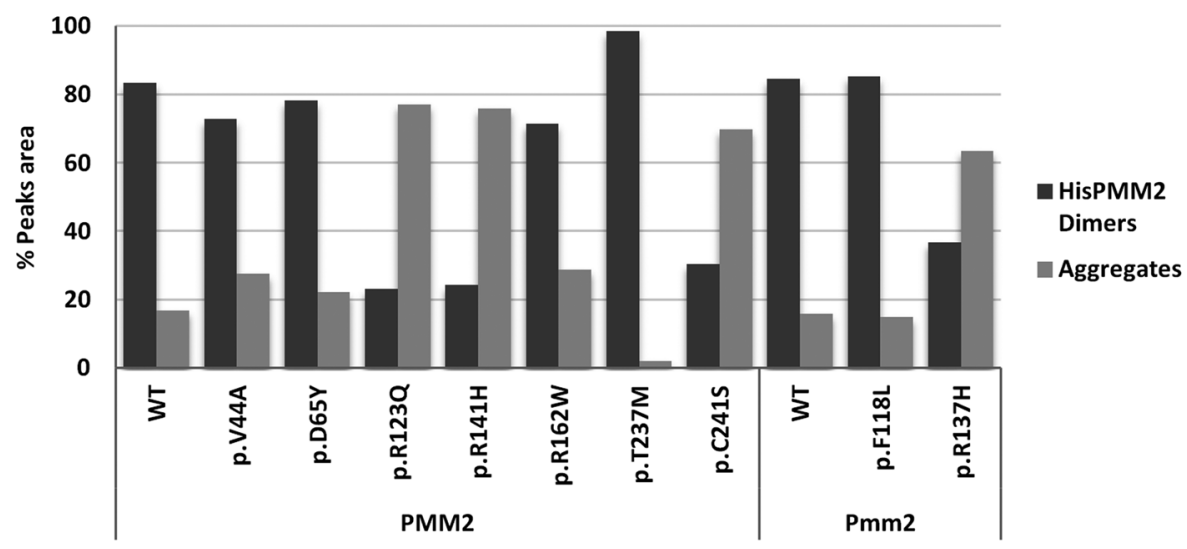

D

PMM2

Pmm2
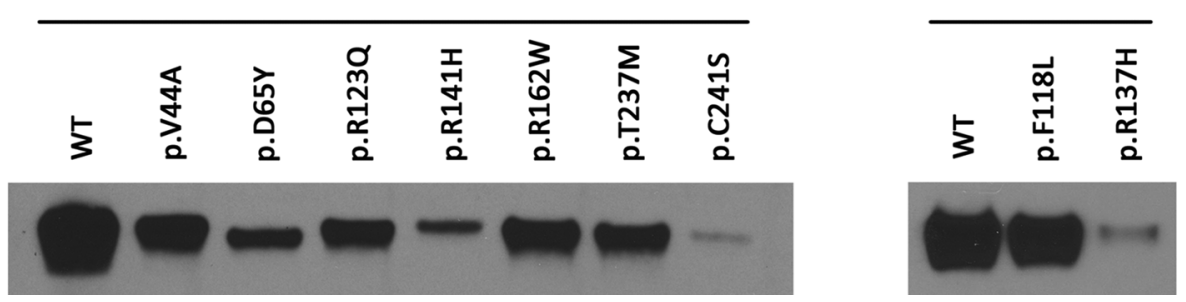

Figure 1. $P M M 2$ mutant oligomerization patterns. A: Size-exclusion chromatograms for the human mutant PMM2 proteins. The gray arrow points to the aggregate peak and the black arrow to the $\mathrm{His}_{6} \mathrm{PMM} 2$ dimer peak. B: Size-exclusion chromatograms for the mutant mouse Pmm2 proteins. C: Percentage of aggregates and $\mathrm{His}_{6} \mathrm{PMM} 2$ dimers for each mutant (except for $\mathrm{p} . \mathrm{F} 207 \mathrm{~S}$ due to its lack of solubility in the bacterial extracts, and p.P113L given the impossibility of obtaining oligomeric fractions). The total amount of purified protein was considered to represent $100 \%$ in each case. D: Western blot of human and mouse pure PMM2/Pmm2 WT and mutant proteins. Equal volumes obtained from the dimer fraction of each protein were loaded onto the SDS-PAGE gel. 
A

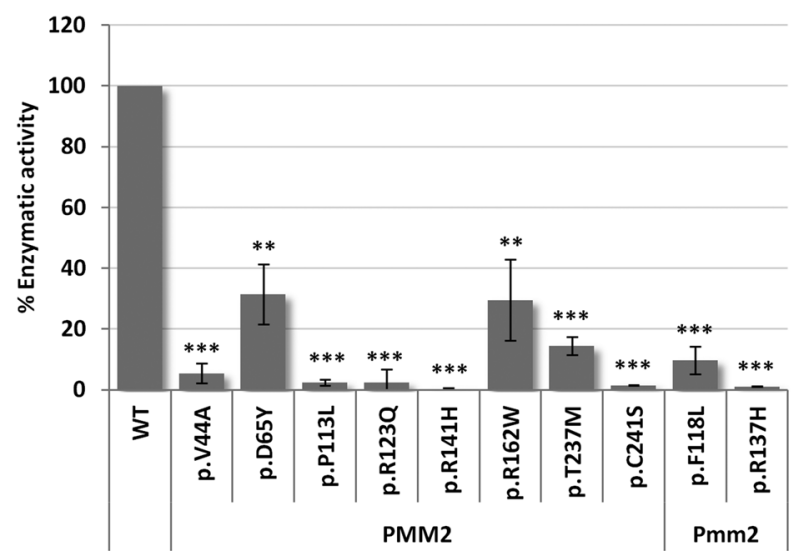

B

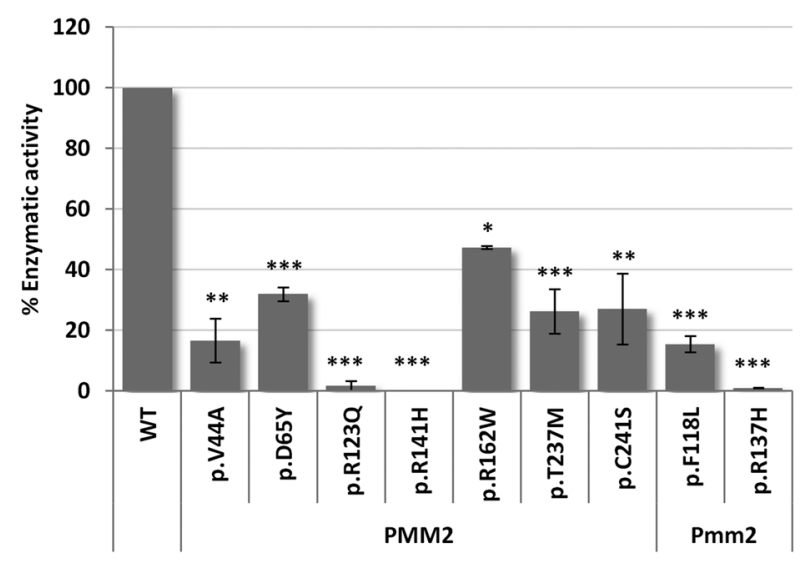

Figure 2. PMM2 activity. A: $\mathrm{His}_{6} \mathrm{PMM} 2 \mathrm{WT}$ and mutant protein activity as determined in bacterial extracts. p.F207S activity could not be determined since it was not expressed in the bacterial extracts. B: Pure dimeric $\mathrm{His}_{6} \mathrm{PMM} 2 \mathrm{WT}$ and mutant protein activity. No oligomeric forms of p.P113L could be obtained; its activity was therefore not determined. WT activity (mU/mg protein) was considered to represent $100 \%$. The results reflect the average of at least three independent experiments $\left({ }^{*} P<0.05 ;{ }^{* *} P<0.01 ;{ }^{* * *} P<0.001\right)$.

It should be noted that none of the oligomerization patterns showed a monomer peak, indicating that PMM2 monomers are likely unstable and tend to aggregate (Fig. 1A).

Some mutants showed a strong tendency to aggregate; for p.R123Q, p.R141H, and p.C241S, the active dimeric forms represented less than half of the purified protein (Fig. 1A and C; Table 1).

The amount of the p.F118L mouse Pmm 2 mutant purified protein was $70 \%$ of the pure WT protein, and showed the same oligomerization pattern, whereas the behavior of $\mathrm{p} . \mathrm{R} 137 \mathrm{H}$ was similar to that of its human analogue p. $\mathrm{R} 141 \mathrm{H}$, showing an oligomerization pattern with an abundance of aggregates (Fig. 1B and C; Table 1).

The purified normal and mutant dimers obtained by gel filtration were analyzed by western blotting (Fig. 1D). Significant differences were observed in the amount of purified protein obtained from most of the mutants compared with the WT; under the same conditions, they were generally much lower for all mutants, although p.F118L mouse Pmm2 production reached some $70 \%$ of the WT total (Table 1).

\section{PMM2 Activity}

PMM2 catalytic activity was analyzed using the soluble bacterial extracts (Fig. 2A) and the pure PMM2 dimer forms of the WT and mutant proteins (Fig. 2B). Remarkable differences were seen in the activity of the WT and mutant proteins, with the activity decay trend similar in both systems. The results obtained with the bacterial extracts showed a lower PMM2 activity in all mutants compared with the WT one $(0.4 \%-31.5 \%$ of the latter's activity) (Fig. 2A; Table 1), whereas for the pure dimeric proteins, the residual activity ranged from $16 \%$ for p.V44A to $47 \%$ for p.R162W. p.D65Y showed $32 \%$ activity compared with the WT, whereas p.T237M and p.C241S showed $26 \%-27 \%$. Almost null catalytic activity was recorded for p.R123Q and p.R141H (Fig. 2B; Table 1). Since p.P113L and p.F207S could not be purified, their activities were not determined. The mouse mutant p.F118L retained $10 \%$ of the Pmm2 WT activity recorded for the bacterial extract, and around 15\% of that recorded for the pure dimeric protein. As seen for human PMM2 p.R141H, p.R137H showed null activity (Fig. 2; Table 1).

\section{PMM2 Stability Analysis}

The thermal stability of the PMM2 mutants was examined by DSF, using the dimeric fraction of the purified proteins (Table 1). Compared with the WT, all the purified human PMM2 mutants showed $T_{\mathrm{m}}$ downshifts of between $0.7^{\circ} \mathrm{C}$ and $10^{\circ} \mathrm{C}$, revealing their intrinsic instability with respect to the WT form. The p.R123Q mutant protein was the most resistant to thermal denaturation, its $T_{\mathrm{m}}$ downshifting by only $0.7^{\circ} \mathrm{C}$ compared with the WT, whereas p.D65Y, with a $T_{\mathrm{m}}$ downshift of $10^{\circ} \mathrm{C}$, was the most sensitive (Fig. 3A; Table 1). The p.F207S and p.P113L mutant proteins could not be analyzed since no dimeric protein could be obtained. The mouse Pmm 2 mutants, p.R137H, showed a downshift of around $4^{\circ} \mathrm{C}$ compared with the WT Pmm2 curve, similar to its human PMM2 analogue p.R141H. In contrast, p.F118L Pmm2 showed no $T_{\mathrm{m}}$ shift, suggesting this mutation does not affect the protein's thermal stability (Fig. 3B; Table 1).

To further investigate the stability of the mutant proteins, time course degradation of WT $\mathrm{His}_{6}$ PMM2 and $\mathrm{His}_{6}$ PMM2 mutant proteins were compared in the prokaryotic transduction-translationcoupled system. PMM2 was immunodetected and the bands quantified by laser densitometry to determine the half-lives (Fig. 4A). Western blotting detected a low molecular weight band that increased in intensity over almost all degradation time courses, further evidencing the unstable nature of the mutations. This band is likely a PMM2 degradation by-product from the $\mathrm{N}$-terminal region since it disappeared in the presence of a His antibody (data not shown).

Human mutant protein half-lives at $37^{\circ} \mathrm{C}$ were reduced by $20 \%-60 \%$ compared with the WT, confirming all the mutants to be more unstable than the WT (Fig. 4C; Table 1). The half-life of p.V44A is not shown since the results obtained were inconsistent (Fig. 4A and C). The half-life of the mouse mutant p.F118L at $37^{\circ} \mathrm{C}$ seemed not to be affected compared with the WT Pmm2 protein (Fig. $4 \mathrm{~B}$ and C). This adds weight to the previous results suggesting that mouse mutation p.F118L does not affect protein stability. It should be noted that the half-life of human PMM2, as determined by this method, is much shorter than that of mouse Pmm2 (around $30 \mathrm{~min}$ for the human protein and $5.4 \mathrm{hr}$ for the mouse protein). 

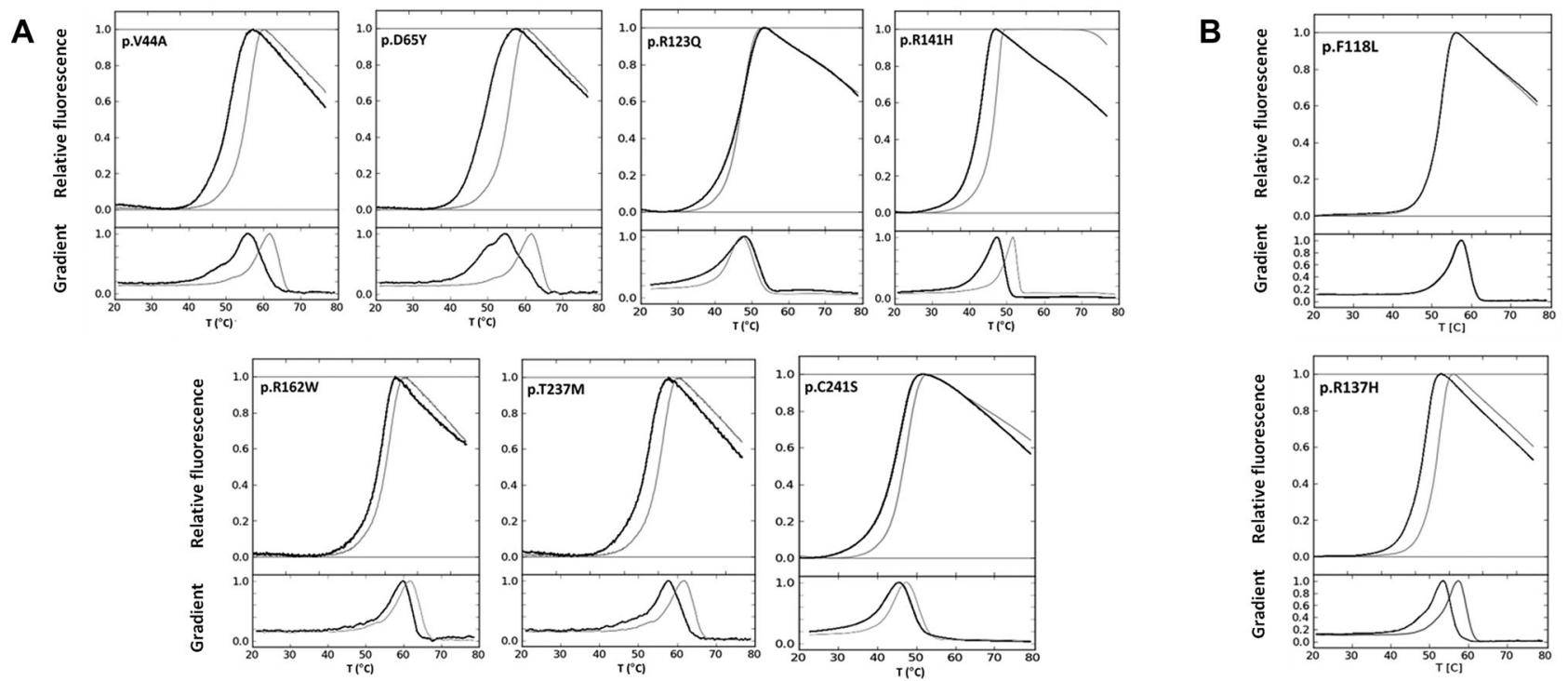

Figure 3. Differential scanning fluorimetry. A: DSF results for the human PMM2 mutants. B: DSF results for the mouse Pmm2 mutants. Representative thermal denaturation profiles for pure mutants (black line) compared with the WT (gray line). The experimental unfolding curves were smoothed, normalized, and analyzed using in-house software. The lower figure in each graph represents the data without scaling.

A

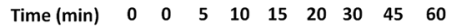

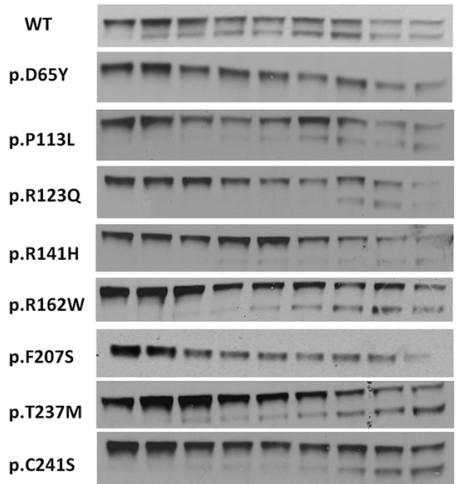

C

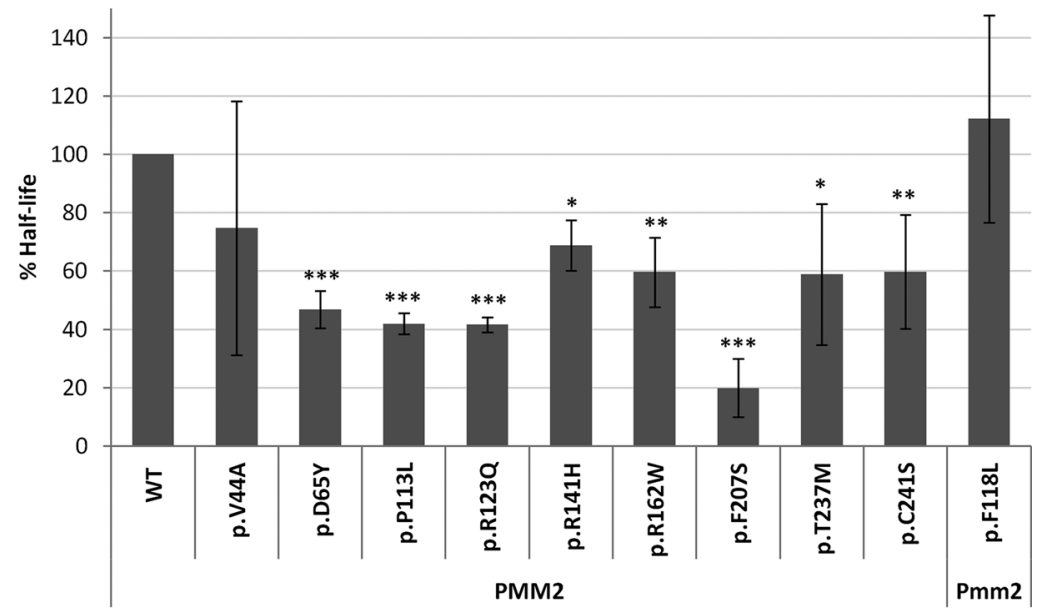

Figure 4. Degradation time courses for PMM2 proteins expressed in the prokaryotic set (transcription-translation-coupled assay). A: Representative western blot of human PMM2 WT and mutant proteins incubated at $37^{\circ} \mathrm{C}$ for $1 \mathrm{hr}$. The half-life of $p . V 44 A$ is not shown due to the inconsistent results obtained. B: Representative western blot of mouse Pmm2 WT and mutant proteins incubated at $37^{\circ} \mathrm{C}$ for $7 \mathrm{hr}$. Equal volumes were loaded onto the SDS-PAGE gel and the protein immunodetected using anti-PMM2 antibodies. Time 0 has been loaded by duplicate. C: Representation of how mutant protein half-lives decrease compared with the WT (considered to reflect $100 \%$ ). The results reflect the average of at least three independent experiments $\left({ }^{*} P<0.05\right.$; $\left.{ }^{* *} P<0.01 ;{ }^{* * *} P<0.001\right)$.

\section{PMM2 Activity and Steady-State Protein Level Under Permissive Conditions in a Eukaryotic System}

PMM2 activity and stability were also studied in a eukaryotic system. Control- and patient-derived fibroblast lines P1 (p.R141H/p.R162W), P2 (c.640-9T>G/p.T237M), P3 (p.Q33*/p. V44A), and P4 (p.R141H/p.D65Y) were transfected with their own folding mutations-p.R162W p.T237M, p.V44A, and p.D65Y, respectively-to increase the amount of protein produced. PMM2 activity assays and western blotting were performed on the soluble part of cell extracts from the patient-derived fibroblast overexpressing the mutants incubated under permissive folding conditions at $28^{\circ} \mathrm{C}$. PMM2 levels and activity were recovered to some extent in all cases (Fig. 5). No increase in activity was seen for the P3 or P4 cells after $45 \mathrm{~min}$ at $37^{\circ} \mathrm{C}$ (unlike for $\mathrm{C}, \mathrm{P} 1$ and $\mathrm{P} 2$ ); the incubation time was therefore increased to $4 \mathrm{hr}$ at which time an increase was observed. 
A

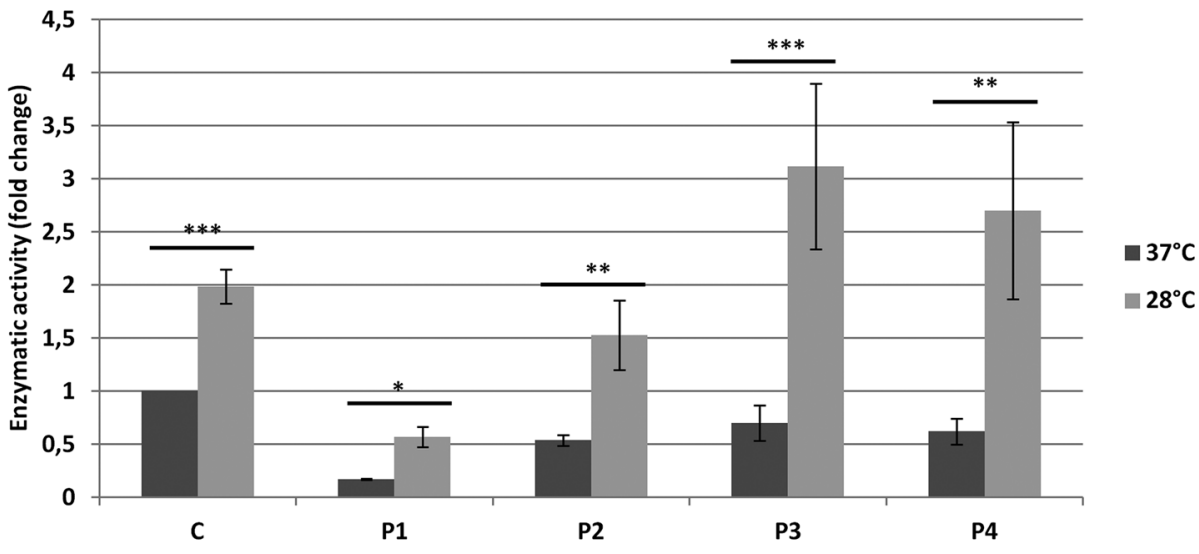

B

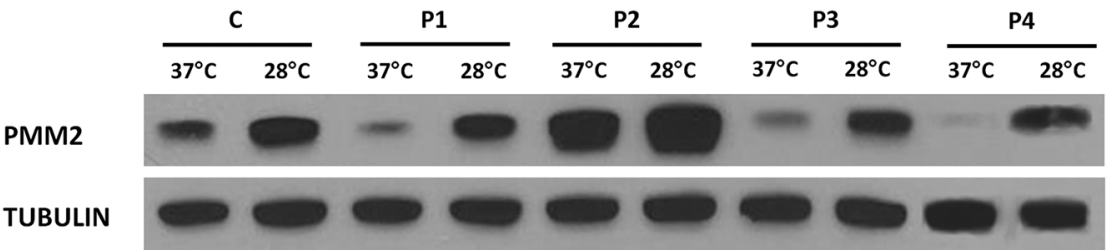

Figure 5. PMM2 activity and steady-state protein level under permissive folding conditions in the eukaryotic system. A: PMM2 activity in the soluble part of the cellular extract from patient-derived fibroblasts stably transfected with their own folding mutations and incubated at $37^{\circ} \mathrm{C}$ or $28^{\circ} \mathrm{C}$. The control PMM2 activity at $37^{\circ} \mathrm{C}$ was taken as 1 . The results reflect the average of at least three independent experiments $\left(^{*} P<\right.$ $\left.0.05 ;{ }^{* *} P<0.01 ;{ }^{* * *} P>0.001\right)$. B: Western blot result for the soluble part of the cellular extract. Cell line genotypes. C: Control overexpressing the WT Flag-PMM2: P1, p.R141H/p.R162W overexpressing the p.R162W mutant; P2, c.640-9T>G/p.T237M overexpressing the p.T237M mutant; P3, p.033X/p.V44A overexpressing the p.V44A mutant; and P4, p.R141H/p.D65Y overexpressing the p.D65Y mutant.

\section{FoldX Prediction Effect on PMM2 Stability}

The computer algorithm FoldX v.3.0 was employed to gain complimentary insight into the effects of PMM2 mutations on protein stability. This algorithm provides a rapid, quantitative estimation of the interactions that contribute toward the stability of proteins. All the mutant proteins, except for p.R162W, seemed to be less stable than the WT; their differences in free energy ranged from 0.22 to $6.51 \mathrm{Kcal} / \mathrm{mol}$ as compared with the WT. p.F207S was the most unstable mutant. These results correlate with the lack of expression of p.F207S in the prokaryotic system. p.R162W appeared to be more stable than the WT, a likely consequence of the in silico nature of the present method (Table 1). Since no crystallized mouse Pmm2 protein was available, predictions for p.F118L Pmm2 were made using its human PMM2 analogue p.F122L. The result showed a difference in free energy with respect to the WT of $0.81 \mathrm{Kcal} / \mathrm{mol}$ (Table 1), indicating the p.F122L PMM2 mutant to be quite unstable. Despite this result, p.F118L Pmm2 instability could not be proved experimentally.

\section{Discussion}

The comprehensive analysis of the mechanisms of action associated with a mutation is an essential step in the development of personalized treatments for patients with a genetic disease. The analysis of these mechanisms also helps to make predictions regarding disease outcome. In the present work, the mechanisms of action of several mutations associated with PMM2-CDG were examined.

The present results show that the mutations p.V44A, p.D65Y, p.R162W, p.R123Q, p.R141H, p.F207S, p.T237M, and p.C241S af- fect the folding properties of the PMM2 protein, whereas p.P113L affects the oligomerization of the PMM2 subunits. Structurally, both mutations p.R123Q and p.R141H also affect the catalytic properties of the protein [Silvaggi et al., 2006; Vega et al., 2011]. The results also highlight the possibility of recovering enzyme activity in the p.V44A, p.D65Y, p.R162W, and p.T237M mutation settings. Steady-state protein levels were recoverable under permissive folding conditions $\left(28^{\circ} \mathrm{C}\right)$ in the eukaryotic expression system.

The pure protein activity results classify the studied mutants into two categories: variants with intermediate residual activity ranging from $16.6 \%$ to $47.3 \%$ (p.V44A, p.D65Y, p.R162W, p.T237M, and p.C241S) that of WT, and variants with null residual activity (p.R123Q and p.R141H) - probably mutations affecting the catalytic site, as indicated by their location within the structure of the protein [Silvaggi et al., 2006; Vega et al., 2011].

Based on the results, we suggest a possible classification of the nine selected mutations as follows: (1) destabilizing mutations (p.V44A, p.D65Y, p.R162W, p.T237M, p.F207S, and p.C241S) that retain some residual activity, (2) folding and functional mutations, affecting both folding and catalytic properties of the PMM2 protein (p.R123Q and p.R141H) that retain null residual activity, and (3) oligomerization mutation affecting PMM2 protein dimerization (p.P113L).

The results obtained by expression and purification of the nine human mutants in the prokaryotic systems, plus the activity assays and degradation time course experiments, the oligomeric and thermal stability profiles, the western blot findings, and the steadystate protein levels studied in the eukaryotic system, suggest that the loss of function of most of these mutant proteins is based on increased susceptibility to degradation and/or aggregation (compared 
with the WT), a common mechanism underlying the so-called conformational diseases caused by protein reduction due to mutants' instability [Gregersen et al., 2006; Muntau et al., 2014; Underhaug et al., 2012].

The degradation of PMM2 proteins harboring missense mutations might be promoted by a folding defect or lie in a problem of oligomeric assembly; certainly, dimer production seems to be essential for the correct functioning of the protein. This work is the first to report an oligomeric profile of PMM2 mutations along with thermal stability data that show the effect of these mutations on the protein's folding properties.

The destabilizing effect of mutations on the PMM2 protein was also made manifest by the significant differences observed in the amount of protein obtained (aggregates/dimers ratio) compared with the WT in crude extracts and pure protein fractions. No pure protein could be obtained for the p.P113L mutation due to its instability. The protein was lost during the purification process but was detected in the crude bacterial extracts.

Most of the mutations associated with PMM2-CDG are missense mutations that cause conformational instability resulting in loss of function [Vega et al., 2011]; however, the present results suggest that the administration of compounds that improve PMM2 folding might provide a treatment. Most PMM2-CDG patients are compound hemizygous with one severe mutation (p.R141H, p.F207S, p.R123Q) and one milder mutation-mostly destabilizing mutations that retain some enzymatic activity [Perez et al., 2011], such as p.V44A, p.D65Y, p.R162W, p.T237M, and p.C241S. Given the high symptomatic threshold of the disease, many patients might benefit from such folding therapy.

One of the aims of the functional mutation analysis is to attempt a genotype-phenotype correlation. The clinical heterogeneity present in PMM2-CDG patients, along with the practical absence of homozygosity among the studied patients, hinders a straightforward genotype-phenotype correlation for this disease. After the analysis of the reported genotypes carrying the studied mutations, we can conclude that just destabilizing mutations retaining residual activity have been detected in homozygosity, such as p.T237M and p.D65Y [Grunewald et al., 2001; Quelhas et al., 2007; Vega et al., 2011]. Furthermore, among the studied mutants, p.R162W and p.T237M have been associated with mild or moderate phenotypes [Grunewald et al., 2001; Quelhas et al., 2007), whereas p.V44A and p.D65Y have been related with more severe phenotypes [Grunewald et al., 2001; Quelhas et al., 2007; Schollen et al., 2007]. This is in agreement with the fact that p.R162W and p.T237M mutations seem to have a milder effect on the PMM2 protein, whereas p.V44A and p.D65Y are both the most unstable ones. Regarding p.C241S mutation, the most stable studied mutant is clearly associated with a milder phenotype [Casado et al., 2012; Giurgea et al., 2005; Grunewald et al., 2001]. Finally, the effect of the p.P113L mutation, originally detected in a homozygous state [Perez et al., 2011; Vega et al., 2011], is, however, not so straightforward. Indeed, its severity seems to have been underestimated as the presented results show the severe effect on the protein.

The possibility of using pharmacological chaperones to treat this disease encouraged the present study in the murine model. The disruption of the mouse $P m m 2$ gene can cause early embryonic lethality [Thiel et al., 2006]. However, the mutations p.R137H and p.F118L are not lethal to embryos if dams are fed mannose during gestation. The effect of these mutations on protein level, catalytic activity, oligomerization profile, and stability revealed the former to behave like its human homologue p. $\mathrm{R} 141 \mathrm{H}$, classifying it as a mutation that affects the stability and catalytic properties of the protein. p.F118L does not affect protein stability, although it does interfere with its catalytic properties. Unfortunately, this mouse model is inadequate for testing pharmacological chaperones or proteostasis regulators since neither mutations cause conformational instability.

In summary, the present results suggest that the destabilizing changes identified in PMM2 might be overcome via the use of tailored pharmacological chaperones. We are currently working to identify such molecules.

\section{Acknowledgment}

We thank Jarl Underhaug of Bergen University, Norway, for his help with the DSF analysis.

\section{References}

Bradford MM. 1976. A rapid and sensitive method for the quantitation of microgram quantities of protein utilizing the principle of protein-dye binding. Anal Biochem 72:248-254.

Briones P, Vilaseca MA, Schollen E, Ferrer I, Maties M, Busquets C, Artuch R, Gort L, Marco M, van Schaftingen E, Matthijs G, Jaeken J, Chabas A. 2002. Biochemical and molecular studies in 26 Spanish patients with congenital disorder of glycosylation type Ia. J Inherit Metab Dis 25:635-646.

Casado M, O'Callaghan MM, Montero R, Perez-Cerda C, Perez B, Briones P, Quintana E, Muchart J, Aracil A, Pineda M, Artuch R. 2012. Mild clinical and biochemical phenotype in two patients with PMM2-CDG (congenital disorder of glycosylation Ia). Cerebellum 11:557-563.

Cline A, Gao N, Flanagan-Steet H, Sharma V, Rosa S, Sonon R, Azadi P, Sadler KC, Freeze HH, Lehrman MA, Steet R. 2012. A zebrafish model of PMM2-CDG reveals altered neurogenesis and a substrate-accumulation mechanism for $\mathrm{N}$ linked glycosylation deficiency. Mol Biol Cell 23:4175-4187.

Cylwik B, Naklicki M, Chrostek L, Gruszewska E. 2013. Congenital disorders of glycosylation. Part I. Defects of protein N-glycosylation. Acta Biochim Polym 60: 151-161.

de Koning TJ, Dorland L, van Diggelen OP, Boonman AM, de Jong GJ, van Noort WL, De Schryver J, Duran M, van den Berg IE, Gerwig GJ, Berger R, Poll-The Bt. 1998. A novel disorder of N-glycosylation due to phosphomannose isomerase deficiency. Biochem Biophys Res Commun 245:38-42.

Eklund EA, Merbouh N, Ichikawa M, Nishikawa A, Clima JM, Dorman JA, Norberg T, Freeze HH. 2005. Hydrophobic Man-1-P derivatives correct abnormal glycosylation in Type I congenital disorder of glycosylation fibroblasts. Glycobiology 15:1084-1093.

Foulquier F, Amyere M, Jaeken J, Zeevaert R, Schollen E, Race V, Bammens R, Morelle W, Rosnoblet C, Legrand D, Demaegd D, Buist N, et al. 2012. TMEM165 deficiency causes a congenital disorder of glycosylation. Am J Hum Genet 91:15-26.

Freeze HH. 2006. Genetic defects in the human glycome. Nat Rev Genet 7:537-551.

Freeze HH. 2009. Towards a therapy for phosphomannomutase 2 deficiency, the defect in CDG-Ia patients. Biochim Biophys Acta 1792:835-840.

Freeze HH, Chong JX, Bamshad MJ, Ng BG. 2014. Solving glycosylation disorders: fundamental approaches reveal complicated pathways. Am J Hum Genet 94:161175.

Giurgea I, Michel A, LeMerrer M, Seta N, de Lonlay P. 2005. Underdiagnosis of mild congenital disorders of glycosylation type Ia. Pediatr Neurol 32:121-123.

Gregersen N, Bross P, Vang S, Christensen JH. 2006. Protein misfolding and human disease. Annu Rev Genomics Hum Genet 7:103-124.

Grunewald S, Schollen E, Van Schaftingen E, Jaeken J, Matthijs G. 2001. High residual activity of PMM2 in patients' fibroblasts: possible pitfall in the diagnosis of CDG-Ia (phosphomannomutase deficiency). Am J Hum Genet 68:347-354.

Hardre R, Khaled A, Willemetz A, Dupre T, Moore S, Gravier-Pelletier C, Le Merrer Y. 2007. Mono, di and tri-mannopyranosyl phosphates as mannose-1-phosphate prodrugs for potential CDG-Ia therapy. Bioorg Med Chem Lett 17:152-155.

Hennet T. 2012. Diseases of glycosylation beyond classical congenital disorders of glycosylation. Biochim Biophys Acta 1820:1306-1317.

Jaeken J, Matthijs G. 2007. Congenital disorders of glycosylation: a rapidly expanding disease family. Annu Rev Genomics Hum Genet 8:261-278.

Kjaergaard S, Skovby F, Schwartz M. 1998. Absence of homozygosity for predominant mutations in PMM2 in Danish patients with carbohydrate-deficient glycoprotein syndrome type 1. Eur J Hum Genet 6:331-336.

Le Bizec C, Vuillaumier-Barrot S, Barnier A, Dupre T, Durand G, Seta N. 2005. A new insight into PMM2 mutations in the French population. Hum Mutat 25:504-505.

Martinez A, Calvo AC, Teigen K, Pey AL. 2008. Rescuing proteins of low kinetic stability by chaperones and natural ligands phenylketonuria, a case study. Prog Mol Biol Transl Sci 83:89-134. 
Matthijs G, Schollen E, Pardon E, Veiga-Da-Cunha M, Jaeken J, Cassiman JJ, Van Schaftingen E. 1997. Mutations in PMM2, a phosphomannomutase gene on chromosome 16p13, in carbohydrate-deficient glycoprotein type I syndrome (Jaeken syndrome). Nat Genet 16:88-92.

Matthijs G, Schollen E, Van Schaftingen E, Cassiman JJ, Jaeken J. 1998. Lack of homozygotes for the most frequent disease allele in carbohydrate-deficient glycoprotein syndrome type 1A. Am J Hum Genet 62:542-550.

Muntau AC, Leandro J, Staudigl M, Mayer F, Gersting SW. 2014. Innovative strategies to treat protein misfolding in inborn errors of metabolism: pharmacological chaperones and proteostasis regulators. J Inherit Metab Dis 37:505-523.

Niesen FH, Berglund H, Vedadi M. 2007. The use of differential scanning fluorimetry to detect ligand interactions that promote protein stability. Nat Protoc 2:2212-2221.

Perez B, Briones P, Quelhas D, Artuch R, Vega AI, Quintana E, Gort L, Ecay MJ, Matthijs G, Ugarte M, Perez-Cerda C. 2011. The molecular landscape of phosphomannose mutase deficiency in iberian peninsula: identification of 15 population-specific mutations. JIMD Rep 1:117-123.

Pey AL, Ying M, Cremades N, Velazquez-Campoy A, Scherer T, Thony B, Sancho J, Martinez A. 2008. Identification of pharmacological chaperones as potential therapeutic agents to treat phenylketonuria. J Clin Invest 118:2858-2867.

Quelhas D, Quental R, Vilarinho L, Amorim A, Azevedo L. 2007. Congenital disorder of glycosylation type Ia: searching for the origin of common mutations in PMM2. Ann Hum Genet 71(Pt 3):348-353.

Richard E, Desviat LR, Ugarte M, Perez B. 2013. Oxidative stress and apoptosis in homocystinuria patients with genetic remethylation defects. J Cell Biochem 114:183191.

Schneider A, Thiel C, Rindermann J, DeRossi C, Popovici D, Hoffmann GF, Grone HJ, Korner C. 2012. Successful prenatal mannose treatment for congenital disorder of glycosylation-Ia in mice. Nat Med 18:71-73.

Schollen E, Keldermans L, Foulquier F, Briones P, Chabas A, Sanchez-Valverde F, Adamowicz M, Pronicka E, Wevers R, Matthijs G. 2007. Characterization of two unusual truncating PMM2 mutations in two CDG-Ia patients. Mol Genet Metab 90:408-413.

Schymkowitz J, Borg J, Stricher F, Nys R, Rousseau F, Serrano L. 2005a. The FoldX web server: an online force field. Nucleic Acids Res 33(Web Server issue):W382-W388.
Schymkowitz JW, Rousseau F, Martins IC, Ferkinghoff-Borg J, Stricher F, Serrano L. 2005b. Prediction of water and metal binding sites and their affinities by using the Fold-X force field. Proc Natl Acad Sci USA 102:10147-10152.

Sharma V, Ichikawa M, Freeze HH. 2014a. Mannose metabolism: more than meets the eye. Biochem Biophys Res Commun 453:220-228.

Sharma V, Nayak J, DeRossi C, Charbono A, Ichikawa M, Ng BG, Grajales-Esquivel E, Srivastava A, Wang L, He P, Scott DA, Russel J et al. 2014b. Mannose supplements induce embryonic lethality and blindness in phosphomannose isomerase hypomorphic mice. FASEB J 28:1854-1869.

Silvaggi NR, Zhang C, Lu Z, Dai J, Dunaway-Mariano D, Allen KN. 2006. The X-ray crystal structures of human alpha-phosphomannomutase 1 reveal the structural basis of congenital disorder of glycosylation type 1a. J Biol Chem 281:1491814926.

Stenson PD, Mort M, Ball EV, Shaw K, Phillips A, Cooper DN. 2014. The Human Gene Mutation Database: building a comprehensive mutation repository for clinical and molecular genetics, diagnostic testing and personalized genomic medicine. Hum Genet 133:1-9.

Studier FW. 2005. Protein production by auto-induction in high density shaking cultures. Protein Expr Purif 41:207-234.

Thiel C, Lubke T, Matthijs G, vonFigura K, Korner C. 2006. Targeted disruption of the mouse phosphomannomutase 2 gene causes early embryonic lethality. Mol Cell Biol 26:5615-5620.

Underhaug J, Aubi O, Martinez A. 2012. Phenylalanine hydroxylase misfolding and pharmacological chaperones. Curr Top Med Chem 12:2534-2545.

Van Schaftingen E, Jaeken J. 1995. Phosphomannomutase deficiency is a cause of carbohydrate-deficient glycoprotein syndrome type I. FEBS Lett 377:318-320.

Vega AI, Perez-Cerda C, Abia D, Gamez A, Briones P, Artuch R, Desviat LR, Ugarte M, Perez B. 2011. Expression analysis revealing destabilizing mutations in phosphomannomutase 2 deficiency (PMM2-CDG): expression analysis of PMM2-CDG mutations. J Inherit Metab Dis 34:929-939.

Westphal V, Kjaergaard S, Schollen E, Martens K, Grunewald S, Schwartz M, Matthijs G, Freeze HH. 2002. A frequent mild mutation in ALG6 may exacerbate the clinical severity of patients with congenital disorder of glycosylation Ia (CDG-Ia) caused by phosphomannomutase deficiency. Hum Mol Genet 11:599-604. 